\title{
Practical Challenges of Enterprise Modeling in the Light of Business and IT Alignment
}

\author{
Julia Kaidalova $^{1}$, Ulf Seigerroth ${ }^{1}$, Tomasz Kaczmarek ${ }^{2}$, and Nikolay Shilov ${ }^{3}$ \\ ${ }^{1}$ School of Engineering, Jönköping University \\ P.O. Box 1026 \\ 55111 Jönköping, Sweden \\ \{julia.kaidalova, ulf.seigerroth\}@jth.hj.se \\ ${ }^{2}$ Poznan University of Economic, Department of Information Systemss \\ Al. Niepodleglosci 10, 61-875 Poznan, Poland \\ t.kaczmarek@kie.ue.poznan.pl \\ ${ }^{3}$ St.Petersburg Institute for Informatics and Automation of the Russian Academy of Sciences \\ 39, 14 Line, 199178, St.Petersburg, Russia \\ nick@iias.spb.su
}

\begin{abstract}
The need to reduce a gap between organizational context and technology within enterprise has been recognized and discussed by both researchers and practitioners. In order to solve this problem it is required to capture and analyze both business and IT dimensions of enterprise operation. In this regard, Enterprise Modeling is currently considered as widely used and powerful tool that enables and facilitates alignment of business with IT. The central role of EM process is EM practitioner - a person who facilitates and drives EM project towards successful achievement of its goals. Conducting EM is a highly collaborative and nontrivial process that requires considerable skills and experience since there are various challenges to manage and to deal with during the whole EM project. Despite quite wide range of related research, the question of EM challenges needs further investigation, in particular concerning the viewpoint of EM practitioners. Thus, the purpose of this paper is to identify challenges that EM practitioners usually face during their modeling efforts taking into consideration potential influence of these challenges on successful conduct of EM and on alignment of Business and IT thereafter.
\end{abstract}

Keywords: Enterprise Modeling, Business and IT Alignment, EM practical challenges.

\section{Introduction}

Successful business management in the dynamically evolving environment demands considerable agility and flexibility from decision makers in order to remain competitive. As a part of business changes and business redesign, there is also a need to have clear understanding about current way of business operation. Stirna and Persson [1] argue that Enterprise Modeling (EM) is one of the most powerful and widely used means that meets both types of needs. They mark out two general 
purposes that EM can be used for. The first purpose is business development, for example, development of business vision and strategies, business operations redesign, development of the supporting information systems, whereas the second one is ensuring business quality, for example, knowledge sharing about business or some aspect of business operation, or decision-making.

EM is a process for creating enterprise models that represent different aspects of enterprise operation, for example, goals, strategies, needs Stirna and Kirikova, [2]. The ability of enterprise models to depict and represent enterprise from several perspectives to provide a multidimensional understanding makes EM a powerful tool that also can be used for Business and IT alignment (BITA) Seigerroth, [3]; Wegmann et al., [4]. In general the problem of BITA has received great attention from both practitioners and researchers Chan and Reich, [5]; Luftman and McLean, [6]. This branch of EM focuses on the gap between the organizational context and technology (information systems in particular) that is pervasive in organization operations and provides a backbone as well as communication means for realizing the organization goals. Particularly, in the domain of modeling similar calls for alignment of information systems and business emerged within various modeling efforts Grant, [7]; Holland and Light, [8]; Seigerroth, [3].

EM is usually a participative and collaborative process, where various stakeholders' points of view are considered and consolidated Stirna and Kirikova, [2]. Two parties of EM are participants from the enterprise itself and EM practitioner (or facilitator) that leads modeling session(s). The first group of stakeholders consists of enterprise employees who have to share and exchange their knowledge about enterprise operations (domain knowledge). There are various factors that can hinder the process of sharing knowledge between enterprise members, for example, as the project progresses the enterprise becomes less interested to allocate their most knowledgeable human resources to modeling sessions, since it can be considered as waste of time Barjis, [9]. The second party of EM is the EM practitioner - a person who facilitates and drives EM project process (partly or fully) towards effectively achieving its goals Persson and Stirna, [10]. This role is responsible for making sure that the project resources are used properly in order to achieve the goals of the project and to complete the project on time ibid, Rosemann et al., [11]. Thus, EM practitioner needs to have considerable experience and broad range of knowledge regarding EM execution, since various problems and challenges occur both during execution of EM sessions and follow-up stages of EM Stirna and Persson, [1].

The need for documentation guidelines related to EM has been revealed and highlighted by several researchers, i.e. cf. Stirna and Persson [1]. Identification of factors that can hinder successful application of EM can be considered as one aspect of such guidelines. Several researchers have claimed that there is a need to investigate challenging factors as an important component of EM practice Bandara et al., [12]; Seigerroth, [3]; Kaczmarek et al., [13]. This has surfaced the need to investigate factors that are considered as challenging from the viewpoint of EM practitioners. In particular, it is interesting to identify challenges that EM practitioner are facing during both EM sessions and the follow-up stages of EM project. Identification and 
description of these challenges can serve as a considerable help for EM practitioners, which can facilitate successful accomplishment of EM project and in turn support BITA within modeled enterprise. The research question of the paper is therefore defined according to below.

\section{What challenges do enterprise modeling practitioners face during EM?}

The rest of the paper is structured in the following way: Section 2 presents related research, Section 3 describes the research method that has been applied to address the research question, in Section 4 and Section 5 results are presented. The paper then ends with c conclusions and discussion of future work in Section 6.

\section{$2 \quad$ Related Research}

A need to deal with a gap between organizational context and technology within enterprise has been recognized and discussed by research community for quite some time Orlikowski and Hofman, [14]. Several researchers have emphasized the need to capture dimensions of both business and IT during design and implementation of IS i.e. cf. Gibson, [15]. In this respect, EM serves as a widely-used and effective practice, because of the core capability of enterprise models to capture different aspects of enterprise operation. Thus, EM currently gets more and more recognition as a tool that can be used for alignment of business with IT Seigerroth, [3].

Performing EM successfully is a nontrivial task that requires considerable skills and experience since there are various issues to manage and to deal with during the whole EM project Stirna et al., [16]. Among core challenges of EM Barjis [9] highlights the complex sociotechnical nature of an enterprise and conflicting descriptions of the business given by different actors.

Indulska et al. [17] present the work that is dedicated to current issues and future challenges of business process modeling with regard to three points of views: academics, practitioners, and tool vendors. The main findings of their work are two lists with top ten items: current business process modeling issues and future business process modeling challenges. They also mention a number of areas that attract attention of practitioners, but still have not been considered by academics, for example, value of business process modeling, expectations management and others. Delen et al. [18] investigates challenges of EM and identified four challenges with regard to decision maker's point of view: heterogeneous methods and tools, model correlation, representation extensibility, and enterprise model compiling.

Another research that investigates the question of EM challenges is presented by Kaczmarek et al. [13]. Their work identifies four challenges of EM, which will serve as a basis for our work. The first challenge is Degree of formalism. There are different modeling notations (from formal machine interpretable languages to very informal rich pictures). The expressivity of the selected formalism impacts the final model. The second one is Degree of detail. Is a problem of deciding how many things need to be put into a model at different layers of EM in order to describe a certain 
situation. The third challenge is Accuracy of the view. It is a challenge of selecting a point of view during modeling. The fourth one is Change and model dependencies. This challenge refers to the fact that modeling is usually done in a constantly changing environment. Models should direct the change in the enterprise, but also models undergo changes. In a multi-layered modeling a change at one layer of the model might has consequences on other layers, and can reflect the change that the enterprise undergoes.

Apart from that, there are several research directions that we consider as related research, below we present three of them. The first are practical guidelines to perform EM. Guidelines are always created in response to challenges and problematic issues that arise during practical activities, therefore it can be possible to get an idea about EM challenges by looking on practical guidelines to perform EM. The second research direction is facets and competence of EM practitioner, which focuses on key factors that determine competence of EM practitioner and highlights, first and foremost, the core questions that EM practitioner is supposed to solve. The third related research direction is EM critical success factors, which focuses on identification of factors that are crucial for success of EM efforts. Since significant part of EM efforts is done by EM practitioner, it is possible to get an idea about EM challenges based on EM critical success factors. Combined overview of these related research directions provided us with a broad foundation regarding potential EM challenges. It helped us on further stages of research, including construction of interview questions and conducting of interviews with respondents.

\subsection{Practical Guidelines to Perform EM}

There are several papers that are introducing different kinds of guidelines for carrying out EM. Stirna et al. [16] describe a set of experiences related to applying EM in different organizational contexts, after what they present a set of generic principles for applying participative EM. Their work marks out five high-level recommendations of using participative EM. Presented generic recommendations are the following: assess the organizational context, assess the problem at hand, assign roles in the modeling process, acquire resources for the project in general and for preparation efforts in particular, conduct modeling sessions.

Stirna and Persson [1] introduce guidelines for carrying out EM in form of antipatterns EM - common and reoccurring pitfalls of EM projects. Presented antipatterns address three aspects of EM - the modeling product, the modeling process, and the modeling tool support. For example, the second group consists of the following anti-patterns: everybody is a facilitator, the facilitator acts as domain expert, concept dump and others. Group addressing EM tool support contains the next issues: models keep "alive" themselves, professionals use only computerized tools, everyone embraces a new tool and others.

\subsection{Facets and Competence of Enterprise Modeling Practitioner}

The significance of the EM practitioner role for overall success of EM project is admitted and discussed by several researchers. Among others, Persson and Stirna [10] 
have presented a work that analyses competence needs for the EM practitioner with regard to different steps in the EM process. They consider that EM process consists of the following activities: project inception and planning, conducting modeling sessions, delivering a result that can be used for subsequent implementation project. Two main competence areas that are identified here are competences related to modeling (ability to model; ability to facilitate a modeling session) and competences related to managing EM projects (for example, ability to select an appropriate EM approach and tailor it in order to fit the situation at hand; ability to interview involved domain experts).

Another view on competence of EM practitioner is presented by Rosemann et al. [11]. They argue that key role of the modeling facilitator has not been researched so far and present a framework that describe four facets (the driving engineer, the driving artist, the catalyzing engineer, and the catalyzing artist) that can be used by EM practitioner.

\subsection{Critical Success Factors}

Critical success factors within the context of EM research can be defined as key factors that ensure the modeling project to progress effectively and complete successfully Bandara et al., [12].

Bandara et al. [12] divide critical success factors of business process modeling into two groups: project-specific factors (stakeholder participation, management support, information resources, project management, modeler experience) and modelingrelated factors (modeling methodology, modeling language, modeling tool).

The work of Rosemann et al. [11] identifies the factors that influence process modeling success. Among them they mention: modeling methodology, modeling language, modeling tool, modelers' expertise, modeling team orientation, project management, user participation, and management support.

\section{Research Method}

General overview of the research path is presented in Figure 1. As a basis for the present work we have used the work of Kaczmarek et al. [13] that is dedicated to multi-layered EM and its challenges in BITA. Our study started from interview design that could fulfill two purposes: validate EM challenges that have been preliminary presented by Kaczmarek et al. [13] and identify other EM challenges. It is important to mention that both kinds of challenges were supposed to be identified considering their potential influence on successful EM execution and, in its turn, on alignment of business and IT subsequently. The next stage included selection of respondents, after what it was possible to conduct interviews. Then collected empirical data has been analyzed, after what it was possible to generate the results in order to answer research question. 


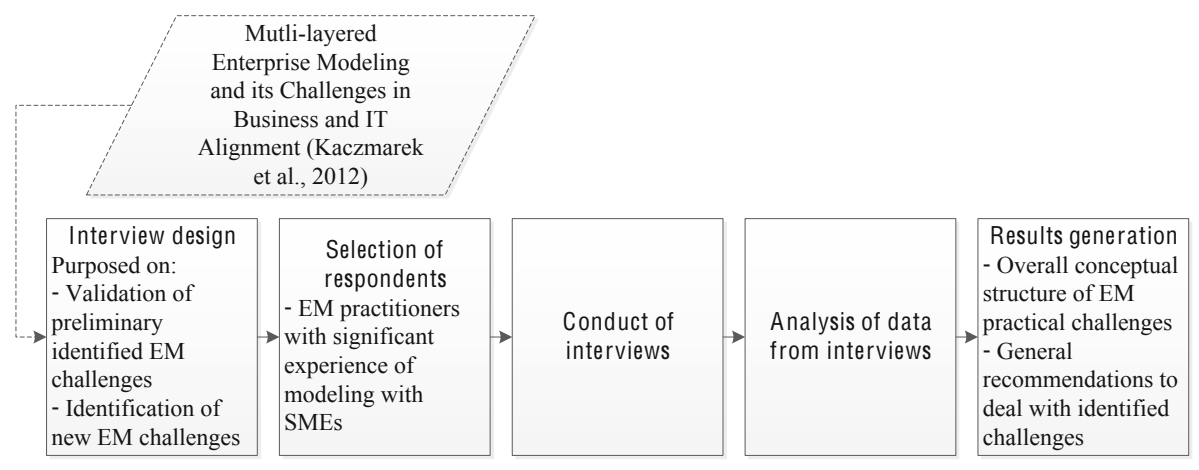

Fig. 1. General research path

\subsection{Interview Design}

In order to identify practical challenges that EM practitioners face it was decided to conduct semi-structured interviews. This kind of empirical research strategy is able to provide in-depth insight into practice of EM and, what is even more important, it allows steering respondents into desired direction in order to receive rich and detailed feedback.

Interview questions consisted of two parts that could provide investigation of EM challenges that have a potential to influence BITA of modeled enterprise: questions with a purpose to identify challenges that EM practitioners face and questions with a purpose to validate preliminary set of EM challenges identified in Kaczmarek et al., [13]. In combination these two groups of questions were supposed to provide comprehensive and integral picture of EM practical challenges. Questions were constructed in such a way that it was possible to identify challenges in both direct and indirect ways. Except from a few examples below the full list of questions can be accessed for download'.

The first part of the interviews had the intention to disclose the most significant challenges that respondents face during EM. In order to carry out this part of interview we have designed a set of direct questions (among others, "When creating enterprise models what challenges you usually face?"). The second group of questions had particular intention to validate preliminary set of EM challenges. This group included both direct and indirect questions. For example, validation of Degree of Formalism challenge has been done with the help of direct question ("Do you consider degree of formalism as a challenging factor?") and a number of indirect questions (among others, "Have you faced the situation when chosen formalism degree was not suitable for further improvement or use of model?"). Having these two types of questions helped us to look into the real fact of the matter instead of just checking it superficially. It should be noted that during further analysis of respondents' answers we were taking into consideration both direct and indirect answers regarding one or another challenge. In other words, challenge was considered

\footnotetext{
${ }^{1}$ http: //hem.hj.se/ kaijul/PoEM2012/
} 
as admitted by particular respondent even if he/she admit it only during answers on indirect questions.

The final question of interviews has been designed in such a way that we could conclude the discussion and get filtered and condensed view on EM practical challenges ("What challenges of EM practice would you consider as a top three?"). An intention here was to make respondents to reconsider and rank the challenges that they have just mentioned, so that it is possible to see which of those they consider as the most important.

\subsection{Selection of Respondents}

Since we have chosen interviews as an empirical method of our work a significant part of the work was dedicated to choosing the right respondents. It was important to find people with considerable EM experience within SMEs. Finally, four respondents with 10-16 years of EM experience have been chosen. Chosen EM practitioners have mostly been working with SMEs within Sweden: Respondent 1 (Managing partner Skye AB), Respondent 2 (Test Manager at The Swedish Board of Agriculture), Respondent 3 (Senior Enterprise Architect at Enterprise Design, Ferrologic AB), and Respondent 4 (Senior Business Consultant at Department for Enterprise Design Ferrologic AB).

\subsection{Conduct of Interviews}

Interviews started from a preliminary stage during which respondents have been provided with brief description of previously identified EM challenges in work of Kaczmarek et al., [13]. This stage had a goal to start and facilitate further discussion by either admitting or denying identified challenges. It also served as a warm-up that opens the main part of the interview, which came right after. The rest of interviews consisted in discussion of prepared question in a very open-ended manner. In other words, respondents were able to build their answers and argumentation quite freely and unconstrained, however, prepared interview questions served as a directive frame for our conversation.

\subsection{Analysis of Interview Data and Results Generation}

Interviews have been recorded and analyzed afterwards. During analysis of interview data our goal was to detect all challenges that have been mentioned by interview respondents, but, what is even more important, it was necessary to logically group detected challenges. This was done by documenting mentioned challenges in a structured manner and putting those challenges, which were related to each other, into one coherent category. Thus, it was possible to generate the main part of results: a set of conceptually structured EM practical challenges. Moreover, we could introduce another part of results, which are general recommendations to deal with presented challenges. However, it is important to make clear differentiation between two deliverables of the present study, since the way to obtain the general structure of EM practical challenges (analysis of interview data as such) differs from the way to obtain 
general recommendations to deal with those challenges (analysis of generated challenges taking into consideration interview data). Results of interview study are presented in the next section.

\section{$4 \quad$ Results of Interview Study}

As it would be expected, EM practitioners are facing various challenges during EM. Several statements of respondents helped us to identify two central activities that unite these challenges (c.f. Figure 2 below).

"On the second phase the most important thing is to translate findings that we have into models, because otherwise it is just a lot of information." (Respondent 1)

"Creating models it is just me sitting in the room with computer and capturing what workshops has concluded... In the workshop situation the most challenging are political aspect and human aspect. These aspects are the most difficult in modeling work." (Respondent 3)



Fig. 2. Two challenging activities of EM

Thus, it was possible to distinguish the first challenging activity, which is extracting information about enterprise by EM practitioner, from the second one, which is further transformation of this information into enterprise models. Interestingly enough, two out of four respondents have strongly emphasized the importance and complexity of the first activity, not the second one.

"Of course there are challenges with actual generation of the model in a tool, but those challenges together are much smaller than challenges with getting the knowledge and information that you need in order to make the model!" (Respondent 3)

"Human-related issues are underestimated! It is people that we are working with. We create models, we build models and we can be very specific about relations between them, but that is just technical stuff. The important thing is to get people that have the right knowledge." (Respondent 4)

Below we present detailed description of challenges that have been identified. In order to generate presented items we considered and, if possible, grouped all challenges that have been mentioned by interview respondents. Statements of interview respondents that we relied on when identifying and generating EM challenges are available for download ${ }^{2}$.

\subsection{Challenges That Are Related to Extracting Information about Enterprise}

This group includes challenges that EM practitioner face while obtaining information about enterprise operation during EM workshops and other fact-finding activities.

${ }^{2}$ http://hem.hj.se/ kaijul/PoEM2012/ 


\section{Right Information}

This challenge is related to the fact that it is usually quite problematic to get information that is really relevant for solving particular modeling problem. According to our respondents, quite often they need to be very persistent and astute while communicating with enterprise employees in order to make them share their knowledge about enterprise operation. Often it leads to the situation when EM practitioner finally has too much information, with different degree of validity and accuracy. The answers also indicate the problem of fuzziness of information, white spots that the participants don't know about and possible inaccuracies in the information obtained from them. This might pose the challenge for modeling which typically requires accurate, complete and clear information.

\section{Group Dynamic and Human Behavior}

Another challenge is that EM practitioner is supposed to deal with group of people that have various tempers, models of behavior and, what is even more important, relations between them. It undoubtedly leads to building unique group dynamic that has to be considered and controlled by EM practitioner in order to steer modeling sessions efficiently.

\section{Shared Language and Terminology}

During EM project different stakeholders usually have different background and consequently different understanding of used terms and relations between these terms. It leads to various problems during EM sessions when stakeholders use different names to address the same concept or, on the contrary, the same names when talking about totally different things. In addition, in some cases employees of an enterprise use some unique terminology that EM practitioner is not familiar with, so EM practitioner needs to adapt in-flight. All these factors lead to the strong need to create shared terminology between project stakeholders in order to create a common ground for efficient communication.

\section{The Purpose of EM and Roles of Stakeholders within It}

One of the most problematic issues during EM project is to make project stakeholders understand the essence of EM as such, since in most of the cases they are not familiar with executive details of EM and with idea of EM in general. Clarification of it might include different aspect: general enlightenment of purposes and goals of EM project; description of roles and relevant responsibilities that different stakeholders are supposed to have within EM project together with description of EM practitioner role; explanation of key capabilities of enterprise models, for example, difference between enterprise models and other representative artifacts.

\subsection{Challenges That Are Related to Transforming Information into Enterprise Models}

This area includes challenges that EM practitioner face while transforming information about enterprise operation into enterprise models. In contrast to the process of obtaining information, this process mostly does not involve collaboration 
of EM practitioner with other stakeholders. It is a process of enterprise models creation in some tangible or intangible form, so that it will be possible to use them further.

\section{Degree of Formalism}

This challenge is related to degree of formalism that is supposed to be used during whole EM project, since existing modeling notations vary from very formal machine interpretable languages to very informal with quite rich pictures (when EM practitioner decides how to document different kinds of findings). From one point of view, it is preferable to use quite formal notation, since in this way enterprise models can be used and reused further even during other projects. However, using formal notation with some stakeholders can hinder the process of modeling, since they might become overloaded and stressed by describing enterprise operations in a way that is too formal for them. Thus, the choice of formalism degree is a quite challenging task that EM practitioner is supposed to solve.

\section{Degree of Detail}

This challenge is about how many details each layer of enterprise model should have. Degree of detail can be high (which includes plenty of details within the model) and low (which includes quite general view on enterprise operation). From one point of view it is important to describe enterprise operation with a high degree of detail, so that it will be possible to see as much elements and interaction between them as possible. However, sometimes it is crucial to have a general view on enterprise functioning, since stakeholders, to the contrary, are interested in rather overall view on it. Thus, the challenge is to leave on enterprise model only important and required details.

\section{Modeling Perspective}

It is a challenge of selecting point of the view during EM. Certainly, enterprise models are able to represent various views on enterprise functioning, which makes them indispensable to deal with different views of stakeholders and with different aspects of enterprise operation. However, in some cases it can be problematic to understand the consequences of adopting certain point of the view on one layer of modeling. In addition, it might be not easy to see how this point of view on one layer will affect other layers.

\section{Change and Model Dependencies}

This challenge is related to the fact that EM is always done in constantly changing environment, which cause the need to keep track of coming changes and update models accordingly. In multi-layered EM it can be quite problematic to keep track of influence of model change on one layer on models on other layers. Some tools enable automatic fulfillment of this task, whereas others do not have such capability.

\section{Scope of the Area for Investigation}

This is a challenge that is related to limiting the scope of the interest during EM. On the one hand, it is important to have rather broad overview of enterprise functioning, 
since it can provide comprehensive and clear view on all actors and cause-effect relationships that take place within modeled enterprise. However, having very broad view can hinder efficient EM, since in this case EM practitioner need to analyze enormous amount of information instead of focusing on the most problematic areas. Thus, it can be quite problematic to define the scope of investigation in properly.

\subsection{Overall Conceptual Structure of EM Practical Challenges}

Taking into consideration interview findings and previous work of Kaczmarek et al. [13], it was possible to build conceptual structure of challenges that EM practitioners face. With the help of interviews it was possible to reveal general conceptual distinction between two challenging areas of EM, that is why it was reasonable to divide EM challenges into two groups. The first group consist of challenges that are related to extraction of information about enterprise, i.e. extract right information, manage group dynamic and human behavior, use shared language and terminology, clarify the purpose of EM and roles of stakeholders within it. The second group consisted of challenges that are related to transforming extracted information into models, i.e. choose degree of formalism, choose degree of detail, adapt modeling perspective, keep track of change and model dependencies, define and stick to the scope of the area for investigation (see Figure 3 below).

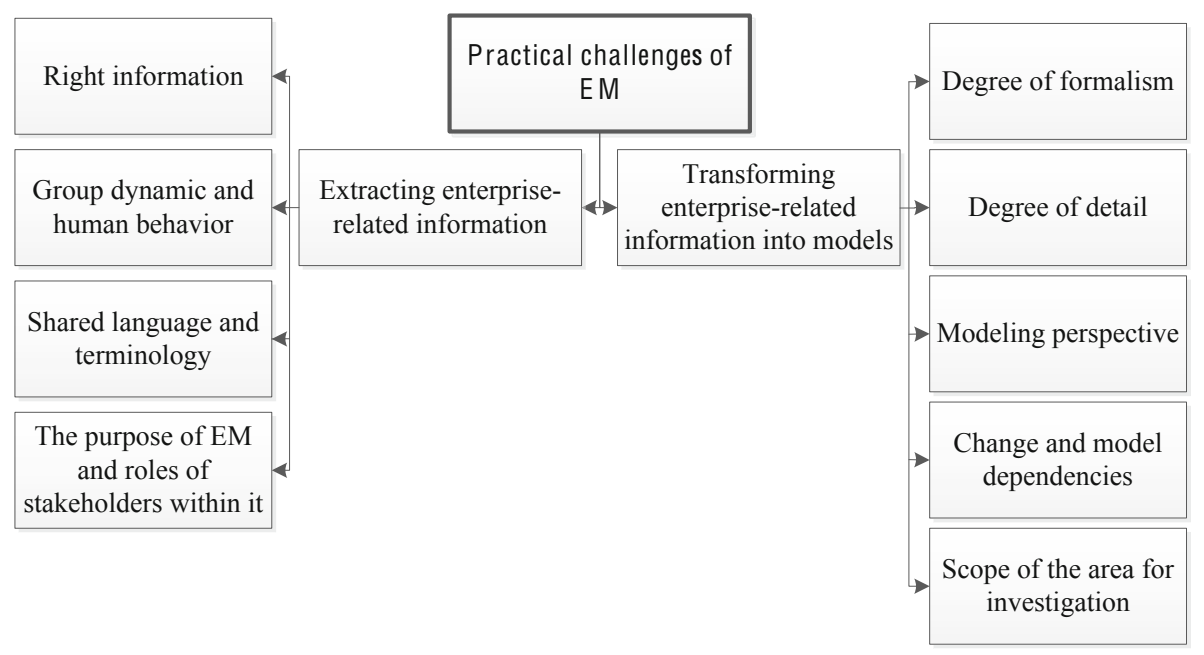

Fig. 3. Overall conceptual structure of EM practical challenges

\section{General Recommendations to Deal with EM Practical Challenges}

In Section 4 we have presented results of performed interview study, which concluded in building conceptual structure of EM practical challenges. Afterwards we could 
analyze created structure of EM challenges, while keeping in mind views and opinions of interview respondents, therefore it was also possible to generate a number of general recommendations that can help EM practitioners to cope with identified EM challenges (see Table 1 below).

General recommendations have been generated taking into consideration opinions of interview respondents. For example, recommendation R15 that can help to cope with degree of formalism challenge is formulated in the following way: "Keep the balance between readability of model and functionality of it depending on the given modeling task and audience". In order to formulate this recommendation we considered statements of Respondent 1 and Respondent 3 ("Sometimes you end up in a need to decide what would be the best: to create good graphical representation or to create sound and valid model. In some cases customers want to generate code from the model, so if the model is inconsistent they definitely get problems with their code generation." Respondent 3; "The problem when you make the model in formal way is that, when you try to describe it, you can really get in trouble with communication and understanding." Respondent 1).

Table 1. EM challenges and general recommendations to deal with them

\begin{tabular}{|c|c|c|}
\hline $\begin{array}{c}\text { Challenge } \\
\text { area }\end{array}$ & Challenge & General recommendations \\
\hline \multirow[t]{4}{*}{$\begin{array}{l}\text { Extracting } \\
\text { enterprise- } \\
\text { related } \\
\text { information }\end{array}$} & $\begin{array}{l}\text { Right } \\
\text { information }\end{array}$ & $\begin{array}{l}\text { R1. Capture what stakeholders know for sure, not what } \\
\text { they believe is true. } \\
\text { R2. Build group of participants for modeling session from } \\
\text { people with relevant knowledge and suitable social skills. }\end{array}$ \\
\hline & $\begin{array}{l}\text { Group } \\
\text { dynamic and } \\
\text { human } \\
\text { behavior }\end{array}$ & $\begin{array}{l}\text { R3. Make everyone involved. } \\
\text { R4. Work with session participants as with group. } \\
\text { R5. Avoid working with too large groups of participants } \\
\text { during EM sessions. } \\
\text { R6. Make sure that you are solving the right task that is } \\
\text { given by right people. }\end{array}$ \\
\hline & $\begin{array}{l}\text { Shared } \\
\text { language and } \\
\text { terminology }\end{array}$ & $\begin{array}{l}\text { R7. Conduct some kind of education (for example, warm- } \\
\text { up introduction as start of modeling sessions). } \\
\text { R8. Depending on audience ground your explanation on } \\
\text { literature, experiences from previous projects or even on } \\
\text { cases from everyday life. } \\
\text { R9. Consider specific terminology that is used by } \\
\text { employees of particular enterprise (for example, some } \\
\text { enterprises have word lists with definitions of key terms). } \\
\text { R10. Use concept model in order to create shared } \\
\text { understanding between EM stakeholders. }\end{array}$ \\
\hline & $\begin{array}{l}\text { The purpose } \\
\text { of EM and } \\
\text { roles of } \\
\text { stakeholders } \\
\text { within it }\end{array}$ & $\begin{array}{l}\text { R11. Make participants understand what model is. } \\
\text { R12. Clarify the role of EM practitioner who is supposed } \\
\text { to lead modeling process. }\end{array}$ \\
\hline
\end{tabular}


Table 1. (coninued)

\begin{tabular}{|c|c|c|}
\hline $\begin{array}{c}\text { Challenge } \\
\text { area }\end{array}$ & Challenge & General recommendations \\
\hline \multirow[t]{5}{*}{$\begin{array}{l}\text { Transforming } \\
\text { enterprise- } \\
\text { related } \\
\text { information } \\
\text { into models }\end{array}$} & $\begin{array}{l}\text { Degree of } \\
\text { formalism }\end{array}$ & $\begin{array}{l}\text { R13. Be consistent with chosen modeling notation } \\
\text { throughout the project, even on conditions that you adjust } \\
\text { formalism degree to the audience. } \\
\text { R14. Avoid being too formal on early stages of EM, since } \\
\text { it can make modeling process too complex for other } \\
\text { participants. } \\
\text { R15. Keep the balance between readability of model and } \\
\text { functionality of it depending on the given modeling task } \\
\text { and audience. }\end{array}$ \\
\hline & $\begin{array}{l}\text { Degree of } \\
\text { detail }\end{array}$ & $\begin{array}{l}\text { R16. Lift a focus if models are unnecessary detailed. } \\
\text { R17. It is usually reasonable to work with different degree } \\
\text { of detail, since often it is important to see business on } \\
\text { different levels. } \\
\text { R18. When communicating with participants it is usually } \\
\text { reasonable to step up from the current level of detail and } \\
\text { start asking WHY question instead of HOW question. } \\
\text { R19. Define the degree of detail on initial stage of EM } \\
\text { taking into consideration goals and purpose of EM project. }\end{array}$ \\
\hline & $\begin{array}{l}\text { Modeling } \\
\text { perspective }\end{array}$ & $\begin{array}{l}\text { R20. Modeling perspectives that you will work with need } \\
\text { to be defined and clarified for other stakeholders on the } \\
\text { initial stage of EM. }\end{array}$ \\
\hline & $\begin{array}{l}\text { Change and } \\
\text { model } \\
\text { dependencies }\end{array}$ & $\begin{array}{l}\text { R21. Use capabilities of CASE-tools that are able to keep } \\
\text { track of relationship and changes between models. } \\
\text { R22. Create enterprise models directly after modeling } \\
\text { sessions, so that information that you have extracted will } \\
\text { not become outdated. }\end{array}$ \\
\hline & $\begin{array}{l}\text { Scope of the } \\
\text { area for } \\
\text { investigation }\end{array}$ & $\begin{array}{l}\text { R23. On the initial stage of EM look at a larger area than } \\
\text { on what stakeholders are describing, however, stay } \\
\text { focused on identified problematic areas during further } \\
\text { stages. }\end{array}$ \\
\hline
\end{tabular}

Another example is recommendation R23 that can deal with challenge of defining the scope of the area for investigation. It is has been formulated considering statements of Respondent 1 and Respondent 2 ("We need to know what we should do and to focus on that." Respondent 1; "If you have a problem and stakeholders think it lies in this area, it is not enough to look at that area, because you need larger picture to really understand the problem. That is why you always need to look at a bigger area in the beginning to get a total picture. It is important that you do not go too narrow too soon!' Respondent 2).

\section{Conclusions and Future Work}

The need to successfully conduct EM in order to align business and IT is acknowledged and discussed, thereby practical challenges of EM are turning out to be an important aspect to investigate. The main purpose of the work was to identify 
challenges that EM practitioners face during EM. Correspondingly, the main finding of the work is a set of conceptually structured practical challenges of EM. It includes two groups of challenges that take place within EM: extracting of information that is related to enterprise operation and transforming this information into models. Challenges that have been discovered within the first activity are right information, group dynamic and human behavior, shared language and terminology, the purpose of EM and roles of stakeholders within it. The second group involves the following challenges: degree of formalism, degree of detail, modeling perspective, change and model dependencies, and scope of the area for investigation. Moreover, work introduced a number of general recommendations that can help EM practitioner to deal with identified challenges.

From practical point of view presented challenges and general recommendations can be considered as supportive guidelines for EM practitioners, which, in its turn, can facilitate successful EM execution and subsequently ensure BITA. From scientific point of view identified challenges and general recommendations can serve as a contribution to the particular areas of EM practical challenges and documented guidelines for conducting EM, which, in a broad sense, makes an input to the question of EM successful execution and, correspondingly, to the question of BITA.

The study has several limitations, which we plan to address in future research. One of them is related to the fact that the data collected at this stage of the study was limited to the Swedish context. We plan to validate the results also for other regions. An important aspect of future work is therefore to elaborate created conceptual structure of EM challenges into comprehensive framework with the help of solid empirical contribution from international EM practitioners, since it is interesting to get a broader picture of EM practical challenges taking into consideration international modeling experience. Second, it would be useful to validate the results obtained from our initial group of practitioners with a larger, more diverse group. This is also subject to our future work. Another aspect that should be considered in future is enhancement of recommendations to deal with EM challenges.

Acknowledgements. This work was conducted in the context of a COBIT collaboration project ("Collaborative Business and IT Alignment"), which is financed by Swedish Foundation for International Cooperation in Research and Higher Education. COBIT is an international collaboration project between Jönköping University (Sweden), Poznan University of Economics (Poland) and St. Petersburg Institute for Informatics and Automation (Russia).

We acknowledge Kurt Sandkuhl, Karl Hammar and Banafsheh Khademhosseinieh for their valuable advices and interesting conceptual discussions during the process of paper writing.

\section{References}

[1] Stirna, J., Persson, A.: Anti-patterns as a Means of Focusing on Critical Quality Aspects in Enterprise Modeling. In: Halpin, T., Krogstie, J., Nurcan, S., Proper, E., Schmidt, R., Soffer, P., Ukor, R. (eds.) BPMDS 2009 and EMMSAD 2009. LNBIP, vol. 29, pp. 407418. Springer, Heidelberg (2009) 
[2] Stirna, J., Kirikova, M.: Integrating Agile Modeling with Participative Enterprise Modeling. In: The Proceedings of the CAiSE Workshop EMMSAD, pp. 171-184 (2008)

[3] Seigerroth, U.: Enterprise Modelling and Enterprise Architecture: the constituents of transformation and alignment of Business and IT. International Journal of IT/Business Alignment and Governance (IJITBAG) 2 (2011) ISSN 1947-9611

[4] Wegmann, A., Regev, R., Loison, B.: Business and IT Alignment with SEAM, REBNITA, RE05, France (2005)

[5] Chan, Y.E., Reich, B.H.: IT alignment: what have we learned? Journal of Information Technology 22, 297-315 (2007)

[6] Luftman, J., McLean, E.R.: Key issues for IT executives. MIS Quarterly Executive 3(2), 89-104 (2004)

[7] Grant, G.G.: Strategic alignment and enterprise systems implementation: the case of Metalco. Journal of Information Technology 18, 159-175 (2003)

[8] Holland, C.P., Light, B.: A Framework for Understanding Success and Failure in Enterprise Resource Planning System Implementation. In: Shanks, G., Seddon, P.B., Willcocks, L.P. (eds.) Second-Wave Enterprise Resource Planning Systems Implementing for Effectiveness, pp. 180-195. Cambridge University Press (2003)

[9] Barjis, J.: Collaborative, Participative and Interactive Enterprise Modeling. In: Filipe, J., Cordeiro, J. (eds.) ICEIS 2009. LNBIP, vol. 24, pp. 651-662. Springer, Heidelberg (2009)

[10] Persson, A., Stirna, J.: Towards Defining a Competence Profile for the Enterprise Modeling Practitioner. In: van Bommel, P., Hoppenbrouwers, S., Overbeek, S., Proper, E., Barjis, J. (eds.) PoEM 2010. LNBIP, vol. 68, pp. 232-245. Springer, Heidelberg (2010)

[11] Rosemann, M., Lind, M., Hjalmarsson, A., Recker, J.: Four facets of a process modeling facilitator. In: Thirty Second International Conference on Information Systems, Shanghai (2011)

[12] Bandara, W., Gable, G.G., Rosemann, M.: Factors and Measures of Business Process Modelling: Model Building Through a Multiple Case Study. European Journal of Information Systems 4(14), 347-360 (2005)

[13] Kaczmarek, T., Seigerroth, U., Shilov, N.: Multi-layered enterprise modeling and its challenges in business and IT alignment. International Conference on Enterprise Information Systems (2012)

[14] Orlikowski, W.J., Hofman, J.D.: An improvisational model for change management: the case of groupware. Sloan Management Science 38(2), 11-21 (1997)

[15] Gibson, C.F.: IT-enabled Business Change: An Approach to Understanding and Managing Risk. MIS Quarterly Executive 2(2) (September 2003)

[16] Stirna, J., Persson, A., Sandkuhl, K.: Participative Enterprise Modeling: Experiences and Recommendations. In: Krogstie, J., Opdahl, A.L., Sindre, G. (eds.) CAiSE 2007. LNCS, vol. 4495, pp. 546-560. Springer, Heidelberg (2007)

[17] Indulska, M., Recker, J., Rosemann, M., Green, P.: Business Process Modeling: Current Issues and Future Challenges. In: van Eck, P., Gordijn, J., Wieringa, R. (eds.) CAiSE 2009. LNCS, vol. 5565, pp. 501-514. Springer, Heidelberg (2009)

[18] Delen, D., Dalal, N.P., Benjamin, P.C.: Integrated modeling: the key to holistic understanding of the enterprise. Communications of the ACM 48(4), 107-112 (2005)

[19] Rosemann, M., Sedera, W., Gable, G.G.: Critical Success Factors of Process Modelling for Enterprise Systems. In: Proceedings of the Americas Conference of Information Systems (AMCIS 2001), Boston, Massachusetts, August 3-5 (2001) 